\title{
Differential autonomic mechanisms underlying early morning and daytime transient myocardial ischaemia in patients with stable coronary artery disease
}

\author{
Ad J van Boven, Jan Brouwer, Harry J G M Crijns, Jaap Haaksma, Kong I Lie
}

\begin{abstract}
Objectives-To see whether autonomic regulatory mechanisms play a part in transient myocardial ischaemia in patients treated with $\beta$ blockers.

Design-Prospective study.

Setting-Outpatients' clinic.

Patients-51 consecutive patients with angiographically documented coronary artery disease, stable angina, and transient myocardial ischaemia despite $\beta$ blockade.

Interventions-24 hour ambulatory electrocardiographic monitoring for analysis of variability in ST depression and heart rate.
\end{abstract}

Main outcome measures-Numbers of episodes of ischaemia, with an ST depression of $\geqslant 0.1 \mathrm{mV} 80 \mathrm{~ms}$ after the $J$ point that lasted $\geqslant 60 \mathrm{~s}$ at an interval of $\geqslant 60 \mathrm{~s}$ from a previous ischaemic episode. Heart rate at onset of ischaemia. Normalised spectral analysis of heart rate variability; ratio of low to high frequency power to assess the sympathovagal balance.

Results-Despite treatment, 258 episodes of transient ischaemia were recorded. At heart rates at onset of ischaemia of $<70$ beats per minute a high ratio of low to high frequency power accompanied the ischaemic events and was paralleled by a remarkably reduced high frequency power. The high ratio-that is, enhanced sympathetic tone during ischaemia-was mainly found in the early morning. By contrast, ischaemic episodes with heart rates at onset of $\geqslant 70$ beats per minute were not associated with significant changes in the parameters of autonomic function.

Conclusions-During $\beta$ blockade the residual transient ischaemia is associated with decreased variability in heart rate. In particular, in ischaemic episodes with a low heart rate at onset the neural regulation of the heart plays a part. Apparently, variability in heart rate is not sufficiently modified by $\beta$ blockers to prevent all ischaemia. The ischaemia related change in the autonomic nervous system during the early morning is in agreement with previous studies, showing increased cardiovascular risk at this time of the day.

(Br Heart f 1995;73:134-138)
Keywords: autonomic nervous system; daytime transient myocardial ischaemia; coronary artery disease

Studies in animals and humans have shown that the autonomic nervous system is important for maintaining coronary vascular tone. ${ }^{1}$ In coronary artery disease a change in the functioning of the autonomic nervous system may therefore play a part in precipitating myocardial ischaemic events. The circadian variation of these events is typified by a higher incidence in the early morning, which may be related to an increase in coronary tone. At this time of the day other factors such as increased platelet aggregability, increase in concentrations of vasoactive hormones, and decreased fibrinolytic activity also may play a part. ${ }^{2-5}$ Obviously, $\beta$ blockade may change the neural regulation underlying these circadian phenomena. ${ }^{6}$ Apart from a beneficial effect of $\beta$ blockers on transient myocardial ischaemia, recent reports suggest that these drugs facilitate ischaemia at low heart rates. ${ }^{7}$

The neural regulation of the heart can be studied by measuring heart rate variability in a 24 hour Holter recording. ${ }^{8}$ The spontaneous beat to beat changes in the electrocardiographic $R R$ intervals are studied with spectral analysis. Vagal efferent activity has been interpreted as being primarily responsible for the high frequency component of the spectrum. ${ }^{9}$ The low frequency component is considered to be related to both vagal and sympathetic outflow. ${ }^{10}$ Vagal and sympathetic activity may act reciprocally, but also in a non-reciprocal way. ${ }^{11}$ The ratio of low to high frequency power of this spectral analysis has been reported to reflect a balance between both autonomic influences, which has been validated by tilt testing and controlled respiration. ${ }^{12}$ Holter monitoring might help to elucidate autonomic mechanisms underlying transient myocardial ischaemia.

We investigated the relation between the circadian variation in functioning of the autonomic nervous system and the occurrence of transient myocardial ischaemia in patients with stable coronary artery disease who had been treated with $\beta$ blockers.

Patients and methods

Ambulatory electrocardiographic monitoring was performed in consecutive patients from the outpatients' clinic who were taking a cardioselective $\beta$ blocker for stable angina pectoris. All had significant coronary artery 
disease, which was defined as a reduction in vessel diameter of at least $50 \%$ on coronary angiography. Patients with left ventricular dysfunction (left ventricular ejection fraction $<30 \%$ ) were excluded. Episodes were classified as ischaemic when an ST segment depression of $\geqslant 0.1 \mathrm{mV}$ lasting for $\geqslant 60 \mathrm{~s}$ occurred $80 \mathrm{~ms}$ after the J point and was separated by $\geqslant 60 \mathrm{~s}$ from a previous ischaemic episode. The time of occurrence and heart rate at the onset of ischaemia were noted for each episode. The median heart rate at the onset of ischaemia served as the cut off point to create two groups of ischaemic episodes in order to estimate whether impaired supply or increased demand of nutrients was the cause of myocardial ischaemia. Ventricular tachycardia was defined as a run of three or more ventricular ectopic beats with a rate of $>100$ beats per minute.

After RR intervals containing ectopic beats were discounted, spectral analysis of heart rate variability was performed using the Marquette 001A program (Marquette Laser System, Milwaukee, Wisconsin, USA). This software computes heart rate variability using $R R$ intervals of consecutive two minute segments and produces average values for each hour as well as a mean of 24 hour values. In this program total frequency was defined as the spectral power between $0.01 \mathrm{~Hz}$ and 1.00 $\mathrm{Hz}$, low frequency as that between $0.04 \mathrm{~Hz}$ and $0 \cdot 15 \mathrm{~Hz}$, and high frequency (HF) as that between $0 \cdot 15 \mathrm{~Hz}$ and $0.40 \mathrm{~Hz}$. We used power units in $\mathrm{ms}^{2}$ after which normalised units for this frequency power was obtained by dividing high frequency by total frequency and low frequency by total frequency. Unreliable spectral curves in periods of distortion were not included in the study. The ratio of sympathovagal balance was defined as the low frequency power divided by high frequency power. To assess the relation between heart rate variability and transient ischaemia spectral analysis of heart rate variability was performed in the hour in which the ischaemic episode occurred and in the hour afterwards. The circadian variation of heart rate variability in relation to the occurrence of ischaemia was assessed by plotting hourly values of the ratio of low to high frequency.

\section{STATISTICAL ANALYSIS}

Heart rate variability parameters during ischaemia were considered to be independent variables. Average heart rate variabilities for the separate episodes are presented in relation to 24 hour means per patient. Owing to the unequal distribution of ischaemic episodes among the patients, no attempt was made to analyse the data on a per patient basis. A nonpaired $t$ test was used to compare the normally distributed heart rate variability parameters during ischaemic events with a heart rate onset of below and above the median. Circadian variation in the hourly values for heart rate variability was tested by repeated measures analysis of variance. Data are presented as means (SE). Differences were considered significant when a $P$ value of less than 0.05 was observed.

\section{Results}

Heart rate variability was assessed in 51 male patients who had transient ischaemia during ambulatory electrocardiographic monitoring while taking a $\beta$ blocker. Forty one patients were taking metoprolol (50-200 mg); 10 used other cardioselective $\beta$ blockers (bisoprolol, atenolol). Their mean age was 60 years; 21 $(41 \%)$ of them had had a myocardial infarction. Two patients used digoxin and $29 \mathrm{cal}-$ cium antagonists. Sixteen patients had silent myocardial ischaemia and ventricular tachycardias.

Despite treatment, 258 episodes of transient ischaemia were noted, which were all asymptomatic. The median number of ischaemic episodes per patient was 3 (range 1-28). The incidence of ischaemia rose in the early morning, and ischaemia was completely absent between 0100 and 0400 .

\section{HEART RATE VARIABILITY}

Spectral analyses are depicted in figures 1 and 2 . During ischaemia total power and high frequency power were reduced compared with 24 hour means. A subsequent rise in all components of spectral power was seen after ischaemia (figure 1). After normalisationthat is, through correction of changes in total power-the high frequency power appeared low during ischaemia and thereafter. The ratio of low to high frequency power as well as

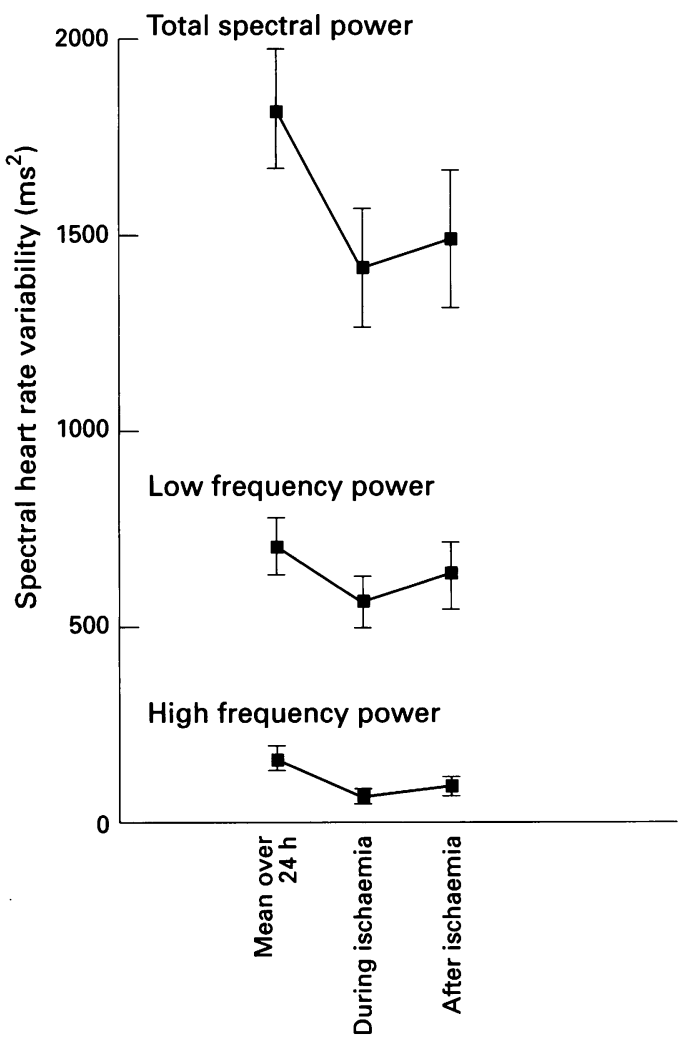

Figure 1 Spectral heart rate variability parameters in 258 transient ischaemic episodes in 51 patients taking $\beta$ blockers. Note the decrease in all powers during ischaemia, suggesting a change in heart rate variability. 
Figure 2 Normalised spectral powers of low and high frequency in normalised units and their ratio. Note the high ratio of low to high frequency power and low normalised high frequency during and after ischaemia, suggesting a withdrawal of vagal tone with reciprocal sympathetic activation.
Figure 3 Heart rate at onset of ischaemia and normalised spectral parameters of heart rate variability in 24 hours during and after transient ischaemia. Note the higher ratio and the lower high frequency at heart rates at onset lower than the median of 70 beats per minute, compared with these parameters during ischaemia at heart rates above 70 beats per minute suggesting that neural influences are mostly recorded during transient ischaemia at low heart rates at onset.
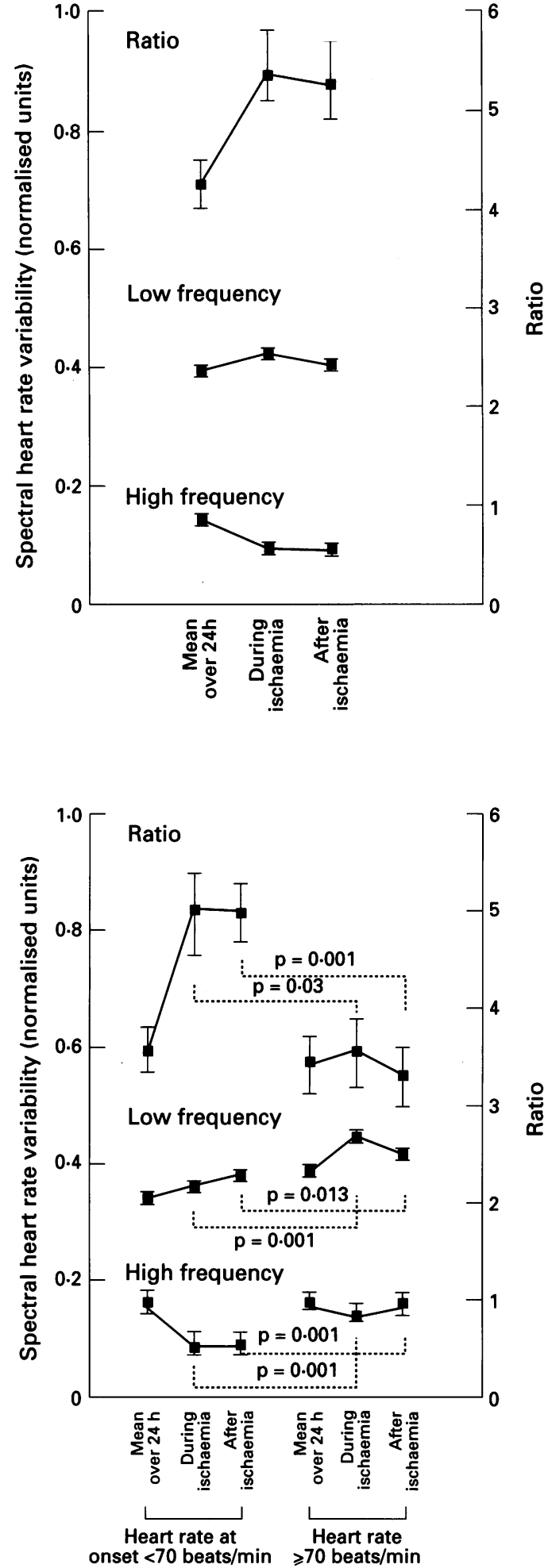

normalised low frequency power increased during ischaemia (figure 2). Median heart rate at the onset of ischaemia was 70 beats per minute. In 109 episodes a heart rate at onset of $<70$ beats per minute was present, and 149 episodes had a heart rate at onset of $\geqslant 70$ beats per minute. The high ratio of low to high frequency power in these patients taking $\beta$ blockers was predominantly found in episodes with a heart rate at onset of ischaemia below 70 beats per minute. In episodes with an onset heart rate $\geqslant 70$ beats per minute there were no significant changes in the spectral components of heart rate vari- ability or their ratio (figure 3). The duration of ischaemia and the total ischaemic burden showed no consistent relation to heart rate variability parameters.

\section{CIRCADIAN PATTERN OF HOURLY HEART RATE} VARIABILITY

The day to night variation in the mean hourly ratio of low to high frequency power is illustrated in figure 4 . In the absence of ischaemia, the curve was flat, showing no variation, presumably because of $\beta$ blockade. In the early morning the ratio during ischaemia was higher compared with other hourly values (not significant by repeated measures analysis of variance). During the day the ratio of low to high frequency power varied but not in a specific way. On the Holter recordings no sustained ventricular arrhythmias-that is, lasting longer than $30 \mathrm{~s}$-were seen. The incidence of unsustained ventricular arrhythmias in this group of patients was low. The extent of ischaemia, however, was more severe in patients with unsustained ventricular tachycardias. No direct association in time between silent myocardial ischaemia and ventricular tachycardias was found.

\section{Discussion}

This study shows that transient myocardial ischaemia on ambulatory electrocardiographic monitoring is accompanied by changes in heart rate variability. Nearly all components of the spectrum of the frequency domain diminished during ischaemia (fig 1). After correction for changes in total power, normalised high frequency spectral power was lower on average during ischaemic episodes (figure 2). When heart rate at the onset of ischaemia was taken into consideration, alterations in neural control were most prominent during episodes with an onset frequency below 70 beats per minute. In other words, these episodes seem to be related to parasympathetic withdrawal with subsequent reciprocal augmentation of sympathetic activity. ${ }^{11}$ Alternatively, neural mechanisms do not seem to play an important part in ischaemia associated with higher heart rates at onset.

To our knowledge this is the first study to show decreased normalised high frequency power as a cause for the increase in the ratio of low to high frequency found during ischaemia. It occurred in most episodes and, as mentioned above, mainly represents vagal inhibition. Morikami et al also proposed parasympathetic withdrawal as a mechanism of ischaemia. They performed handgrip testing in patients with variant angina and found a lower vagal activity a few minutes before coronary vasospasm was observed. ${ }^{13}$ By contrast, Bernardi et al found an increase in low frequency power in asymptomatic nocturnal ischaemia. ${ }^{14}$ They did not, however, consider low frequency power and the changes in total power during the day, which precludes a general conclusion about the nature of the change in sympathovagal balance. Our study unifies the contentions of the previous studies, as it 


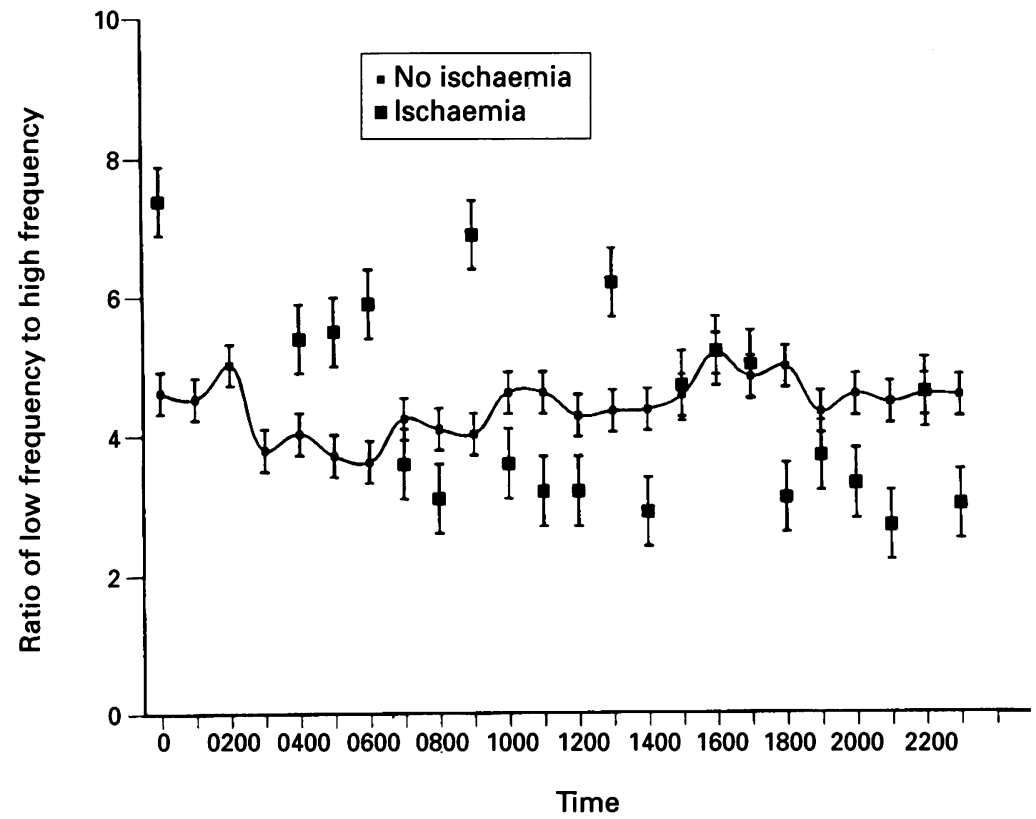

Figure 4 Hourly pattern in ratio of low to high frequency power. In absence of ischaemia there is no day to night variation owing to $\beta$ blockade. During ischaemia there is a large variation in ratio, possibly because of differences in heart rate at onset of ischaemia and physical activity. Note, however, the high early morning ratio, reflecting predominant sympathetic activity. (Not significant by repeated measures analysis of variance.)

shows the permissive role of vagal tone in the adrenergic activation leading to ischaemia. Above all, it underscores and supports previous work on the importance of considering sympathovagal balance rather than sympathetic or parasympathetic activation as both systems work reciprocally as well as in a nonreciprocal way. ${ }^{12}$

\section{MECHANISMS FOR ISCHAEMIA AT LOW HEART RATE}

Holter monitoring has shown that ischaemia can occur at low heart rates. Our results suggest that this type of ischaemia is facilitated by specific neural mechanisms. Obviously, low heart rate ischaemia is not associated with an increased oxygen demand. ${ }^{15}$ Therefore, other mechanisms should be involved. From our study, neural regulation of the vascular tone and subsequent coronary narrowing could be held responsible for this type of ischaemia.

The extent to which $\beta$ blockers can enhance transient ischaemia at low heart rates cannot be estimated in our study. However, an increase in incidence of episodes of ischaemia at low heart rates after $\beta$ blockade was found by McLenachan et al. ${ }^{7}$ In addition, El-Tamimi et al describe an enhanced coronary vasoreactive response to acetylcholine in patients taking $\beta$ blockers. ${ }^{16}$ Despite $\beta$ blockade, a high ratio of low to high frequency power was obtained during ischaemia at low heart rates, which shows sympathetic activity. This finding might imply a cardiac spillover of catecholamines, which can provoke paradoxical narrowing of diseased coronary segments. ${ }^{17} 18$ Our data on the neural regulation of low heart rate ischaemia are compatible with the concept of vasomotor disturbance, in which $\beta$ blockers obviously have an important role.

\section{CIRCADIAN VARIATION}

The circadian rhythm in the ratio of low to high frequency power seen in patients who are not being treated was absent in our patients owing to $\beta$ blockade. ${ }^{6}$ During ischaemia, however, there was a large variation in this ratio (figure 4). We found high values in the early morning, which is in agreement with the increase in sympathetic activity in the early morning reported by Furlan et al. ${ }^{19}$ Using a non-spectral method, Malik et al also found that reduced heart rate variability was most prominent at $0600 .{ }^{20}$ In patients refractory to the anti-ischaemic effects of $\beta$ blockade the sympathovagal balance in the early morning seems to follow a pattern similar to that seen in control patients. ${ }^{6}$

\section{NORMALISATION OF SPECTRAL ANALYSIS}

Owing to a large variation in total spectral power, the spectral heart rate variability parameters might change considerably during the day, interfering with a unified interpretation of the results. Using an instantaneous heart rate function for spectral analysis, Arai et al reported an 18-fold reduction in low frequency power during maximal exercise compared with the resting value. ${ }^{21}$ In concordance with other investigators we presented spectral analysis of heart rate variability in normalised units after modifying the data obtained with the Marquette program. Normalisation of heart rate variability parameters derived from Holter recordings is a prerequisite for the interchangeable use of spectral results.

\section{CLINICAL IMPLICATIONS}

In patients with long lasting ischaemia, extensive coronary artery disease, and an impairment of left ventricular function a permanently decreased high frequency power on a 24 hours electrocardiographic monitoring has been taken to indicate an adverse prognosis. ${ }^{22}{ }^{23}$ Other studies related low heart rate variability parameters to an increased risk for sudden death. ${ }^{24-26} \mathrm{~A}$ low heart rate variability in general has been related to an increased risk for sudden cardiac death. ${ }^{27-29}$ $\beta$ Blockers have been reported to increase mainly the high frequency component of heart rate variability. ${ }^{60-32} \mathrm{~A}$ high ratio of low to high frequency power is therefore not expected. Enhancement of the cardiac vagal tone (high frequency) by $\beta$ blockers can protect against sudden death from arrhythmias. As a consequence, since the high frequency component is decreased, these alleged cardioprotective properties of $\beta$ blockers are temporarily lost during ischaemia.

Despite $\beta$ blockade, 258 episodes of ischaemia could be detected in our population. Complete abolishment of ischaemia on the ambulatory recordings is often difficult to attain. ${ }^{7}$ Since neural regulation seems to be so important, the additional effect on vagal tone of muscarinic blocking agents needs to be evaluated. ${ }^{33}$ They may potentiate the increase in vagal tone realised by $\beta$ blockers, but whether this acts in an anti-ischaemic way remains to be studied. 
CONCLUSIONS

During $\beta$ blockade the residual transient ischaemia is associated with decreased heart rate variability. In particular, in ischaemic episodes with a low heart rate at onset the neural regulation of the heart plays a part. Apparently, heart rate variability is not sufficiently modified by $\beta$ blockers to prevent all ischaemia. The marked ischaemia related change in the autonomic nervous system during the early morning is in agreement with previous studies and shows increased cardiovascular risk at this time of the day.

We thank Professor A Malliani for his valuable comments.

1 Friesinger GC. Silent myocardial ischaemia and the importance of coronary vasoconstriction. Curr Opin Cardiol 1989;4:492-8.

2 Richards AM, Nicholls MG, Espiner EA, Ikram H, Cullens $M$, Hinton D. Diurnal patterns of blood pressure, heart rate and vasoactive hormones in normal man. Clinical and Experimental Theory and Practice [A] 1986;8:153-66.

3 Muller JE, Tofler GH, Stone PH. Circadian variation and triggers of onset of acute cardiovascular disease. Circulation 1989;79:733-43.

4 Muller JE, Ludmer PL, Willich SN, Tofler GH, Aylmer $\mathrm{G}, \mathrm{Klangos} \mathrm{I}$, et al. Circadian variation in the frequency of $\mathrm{G}$, Kangos I, et al. Circadian variation in the freque

5 Turton MB, Deegan T. Circadian variations of plasma catecholamine, cortisol and immunoreactive insulin concatecholamine, cortisol and immunoreactive insulin concentrations in

6 Brouwer J, Portegies MJM, Tuininga YS, van Boven AJ, Haaksma J, vd Ven LLM, et al. Effects of bisoprolol on the circadian distribution of silent myocardial ischaemia and heart rate variability parameters [abstract]. Eur Heart $\mathcal{f} 1993 ; 14$ (suppl):299.

7 McLenachan JM, Weidinger FF, Barry J, Yeung A, Nabel EG, Rocco MB, et al. Relations between heart rate, ischaemia and drug therapy during daily life in patients with coronary artery disease. Circulation 1991;83: 1263-70.

8 Akselrod S, Gordon D, Ubel FA, Shannon DC, Barger AC, Cohen TJ. Power spectrum analysis of heart rate fluctuations: a quantitative probe of beat-to-beat cardiovascular control. Science 1981;213:220-2.

9 Chess GF, Tam RMK, Caleresu FR. Influence of cardiac neural inputs on rhythmic variations of heart period in neural inputs on rhythmic variations of

10 Pomeranz B, Macauly RJB, Caudill MA, Kutz I, Adam D, Gordon $\mathrm{D}$, et al. Assessment of autonomic function in
humans by heart rate spectral analysis. Am 7 Physiol humans by heart

11 Koizomi K, Kollai M. Multiple modes of operation of cardiac autonomic control: development of the ideas from Cannon and Brooks to the present. $\mathcal{f}$ Auton Nerv Syst 1992;41:19-30.

12 Pagani M, Lombardi F, Guzetti S, Rimoldi O, Furlan R, Pizzinelli $\mathrm{P}$, et al. Power spectral analysis of heart rate and arterial pressure variabilities as a marker of sympatho-vagal interaction in man and conscious dog. Circ Res 1986;59:178-93.

13 Morikami Y, Yasue H, Okumura K, Horio Y, Fuji H, Matsuyama $\mathrm{K}$. Effects of phentolamine and atropine on angina pectoris induced by hand grip test in patients angina pectoris induced by hand grip test in

14 Bernardi L, Lumina C, Ferrari MR, Ricordi L, Vandea I, Frattino $\mathrm{P}$, et al. Relationship between fluctuation in heart rate and asymptomatic nocturnal ischaemia. Int $\mathcal{f}$ Cardiol 1988;20:39-51.

15 Cohn PF. Silent myocardial ischaemia and infarction. 2nd ed.: New York: Marcel Dekker, 1989.

16 El-Tamimi H, Mansour M, Wargovich TJ, Hill JA, Mills $\mathrm{RM}$, Nunn C, et al. Beta-blockers enhance coronary vasoreactive response to acetylcholine in patients with stable angina [abstract]. f Am Coll Cardiol 1993;21:19A.

17 Suematsu M, Ito Y, Fukuzaki $H$. The role of parasympathetic nerve activity in the pathogenesis of coronary vasospasm. fpn Heart f 1987;28:649-62.

18 Rozanski A, Bairey CN, Krantz DS, Friedman J, Resser KJ, Morrel M, et al. Mental stress and the induction of silent myocardial ischaemia in patients with coronary artery disease. $N$ Engl $7 \mathrm{Med}$ 1988;318:1005-12.

19 Furlan R, Guzetti S, Crivellaro W, Dassi S, Tinelli M, Cerutti S, et al. Continuous 24-hour assessment of the neural regulation of systemic arterial pressure and $R R$ variabilities in ambulant subjects. Circulation 1990;81: 537-47.

20 Malik M, Farrell T, Camm AJ. Circadian rhythm of heart rate variability after acute myocardial infarction and its
influence on the prognostic value of heart rate variability. influence on the prognostic value

21 Arai Y, Saul JP, Albrecht P, Hartley LH, Lilly LS, Cohen RJ, et al. Modulation of cardiac autonomic activity during and immediately after exercise. Am $\mathcal{F}$ Physiol 1989; 256:H132-41

22 Hayano J, Sakakibara Y, Yamada Y, et al. Decreased magnitude of heart rate spectral components in coronary artery disease: its relation to angiographic severity. Circulation 1990;81:1217-24.

23 Binkley PF, Nunziata E, Haas GJ, Nelson SD, Cody RJ Parasympathetic withdrawal is an integral component of autonomic dysbalance in congestive heart failure: demonstration in human subjects and verification in paced canine model of ventricular failure. $7 \mathrm{Am}$ Coll Cardiol 1991;18:464-72

24 Algra A, Tijssen J, Lubsen J. Heart rate variability and risk for sudden death [abstract]. Circulation 1990;82:III-751.

25 Kleiger RE, Miller JP, Bigger TJ, Moss AJ and the Multicenter Post-infarction Research Group. Decreased heart rate variability and its association with increased mortality after acute myocardial infarction. Am $\mathcal{f}$ Cardiol mortality after acute

26 Myers GA, Martin GJ, Magid NM, Barnett PS, Schaad JW, Weiss JS, et al. Power spectral analysis of heart rate variability in sudden cardiac death: comparison to othe methods. IEEE Trans Biomed Eng 1986;12:1149-56.

27 Martin GJ, Magid NM, Myers G, Barnett PS, Schaad JW Weiss JS, et al. Heart rate variability and sudden death secondary to coronary artery disease during ambulatory electrocardiographic monitoring. Am f Cardiol 1987; 60:86-9.

28 Bigger JT Jr, Fleiss JL, Rolnitzky LM, Steinman RC. Frequency domain measures of heart period variability to assess risk late after myocardial infarction. $7 \mathrm{Am}$ Coll Cardiol 1993;21:729-36.

29 Farrell TG, Bashir Y, Cripps T, Malik M, Poloniecki J, Bennett D, et al. Risk stratification for arrhythmic events in postinfarction patients based on heart rate variability, ambulatory electrocardiographic variables and the signal ambulatory electrocardiographic variables and the signa averaged

30 Cook JR, Bigger JT, Kleiger RE, Fleiss JL, Steinman RC Rolnitzky LM. Effect of atenolol and diltiazem on hear period variability in normal persons. $\mathcal{F} \mathrm{Am}$ Coll Cardiol 1991;17:480-4.

31 Mølgaard H, Mickley H, Pless P, Bjerregaard P, Mølle $M$. Effects of metoprolol on heart rate variability in survivors of acute myocardial infarction. $\mathrm{Am} \mathcal{F}$ Cardiol 1993;71:1357-9.

32 Lambert CR, Coy K, Imperi G, Pepine CJ. Influence of beta adrenergic blockade defined by time series analysis on circadian variation of heart rate and ambulatory myocardial ischaemia. Am $\mathcal{f}$ Cardiol 1989;64:835-9.

33 Casadei B, Pipilis A, Sessa F, Conway J, Sleight P. Low doses of scopolamine increase cardiac vagal tone in the acute phase of myocardial infarction. Circulation 1993; 88:353-7. 